"Buscar la verdad y afianzar los valores transcendentales", misión de las universidades en su artículo primero, inspirado en los principios humanísticos. Ley de Universidades 8 de septiembre de 1970.
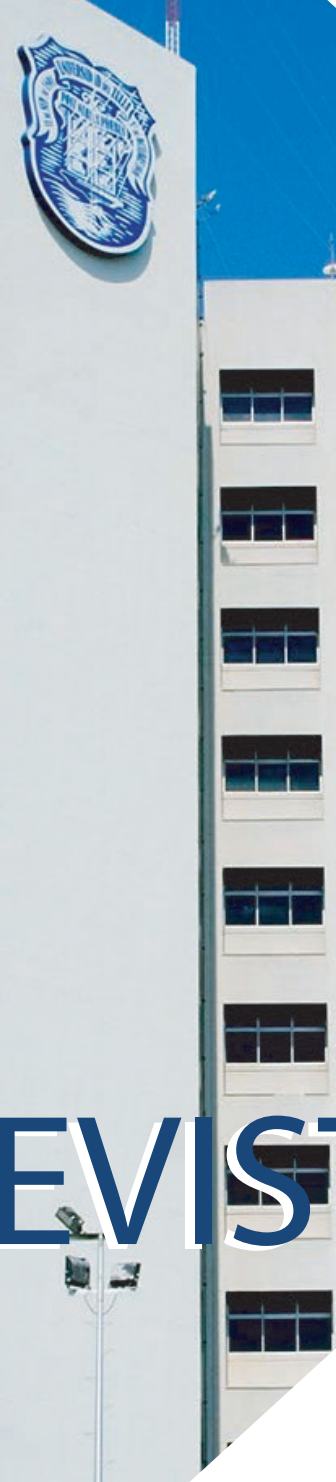
Rev. Téc. Ing. Univ. Zulia. Vol. 43, No. 3, 2020, 114-120

\title{
Termólisis de Clorooxoacetato de Etilo en Fase Gas: Comparación con Oxoacetato de Etilo y Detalles del Sistema Estático para el Estudio Cinético
}

\author{
Andreína Alexandra Reyes Yanes $\mathbb{D}$ \\ Universidad Nacional Mayor de San Marcos, Facultad de Química e Ingeniería Química, Departamento \\ Académico de Fisicoquímica. Ciudad Universitaria- Pabellón B- Calle Germán Amezaga № 375- Lima- Perú \\ 15081. areyesy@unmsm.edu.pe \\ https://doi.org/10.22209/rt.v43n3a01
}

Recepción: 03/03/2020 | Aceptación: 05/06/2020 | Publicación: 01/09/2020

\section{Resumen}

La ventaja de los estudios cinéticos en fase gas es que el coeficiente de velocidad no es afectado por superficies, catalizadores ni disolventes que interactúen con el sustrato. La termólisis en fase gas de los ésteres derivados del ácido oxálico ha sido poco estudiada. En esta investigación se realizó el estudio cinético de la descomposición térmica de clorooxoacetato de etilo entre 543-593 K y 76-209 mbar, utilizando un sistema estático de vacío cuyo funcionamiento se describe ampliamente. Mediciones de presión total y análisis cromatográfico de productos indican reacciones consecutivas de orden uno, unimoleculares y homogéneas, de decarbonilación del sustrato seguida de la eliminación de etileno, a diferencia de la termólisis de oxoacetato de etilo, que procede por vías paralelas de decarboxilación y decarbonilación. La ecuación de Arrhenius para la reacción estudiada entre 543,2 - 593,1 K resultó ser log $\mathrm{k}_{1}=(13,22 \pm 0,45)-(179,4 \pm 4,9) \mathrm{kJ}$ $\mathrm{mol}^{-1}(2,303 \mathrm{RT})^{-1}$, y el coeficiente de velocidad a $623 \mathrm{~K}$ significativamente mayor que para la decarbonilación del oxoacetato de etilo sugiere que el sustituyente cloro tendría mayor disposición que el hidrógeno de migrar al carbonilo adyacente, formando un estado de transición con enlaces más rígidos.

Palabras clave: termólisis; cinética en fase gas; clorooxoacetato de etilo

\section{Gas Phase Ethyl Chlorooxoacetate Thermolysis: Comparison with Ethyl Oxoacetate and Static System Details for Kinetic Study}

\begin{abstract}
The advantage offered by kinetic studies in the gas phase is that the velocity coefficient is not affected by surfaces, catalysts or solvents that interact with the substrate. So far, gas phase thermolysis of esters derived from oxalic acid have been scarcely studied. In this investigation, the kinetic study of the thermal decomposition of ethyl chlorooxoacetate between 543-593 K and 76-209 mbar was carried out, using a static vacuum system whose operation are widely described. Total pressure measurements and chromatographic products analysis indicates consecutive first order reactions, unimolecular and homogeneous for substrate decarbonylation, followed by ethylene elimination, unlike the ethyl oxoacetate thermolysis, which proceeds by parallel decarboxylation and decarbonylation pathways. Arrhenius equation for the reaction studied between $543.2-593.1 \mathrm{~K}$ was found to be $\log \mathrm{k}_{1}=(13.22 \pm 0.45)-(179.4 \pm 4.9) \mathrm{kJ} \mathrm{mol}^{-1}(2.303 \mathrm{RT})^{-1}$ and velocity coefficient at $623 \mathrm{~K}$ significantly larger than for the decarbonylation of ethyl oxoacetate, suggesting that chlorine substituent would have a greater tendency than hydrogen to migrate to the adjacent carbonyl, forming more rigid bonds transition state.
\end{abstract}

Keywords: thermolysis; gas-phase kinetics; decarbonilation 


\section{Introducción}

Las reacciones homogéneas pueden llevarse a cabo tanto en fase gas como en disolución, en este último caso existe una alta probabilidad de que los disolventes, al igual que las superficies y los catalizadores, puedan interactuar con el sustrato y afectar el coeficiente de velocidad. Por esta razón, las reacciones en fase gas no tienen limitaciones para que la obtención de los parámetros cinéticos y el estudio del comportamiento de la molécula aislada en el estado de transición puedan efectuarse con ayuda de modelos mecanocuánticos. Sin embargo, aunque las termólisis en fase gas son importantes por constituir un raro caso de reacciones de orden uno y, adicionalmente, esclarecer la relación estructura- reactividad de ciertos sustratos bajo estudio [1-9], el número de reacciones en fase gas no complejas es relativamente pequeño [10]. Los análogos del ácido oxálico han sido escasamente investigados en fase gas. Un estudio teórico-experimental sobre la termólisis del clorooxoacetato de metilo [11] sugiere el siguiente mecanismo, sustentado por la concordancia entre los valores calculados por métodos semiempíricos PM3 y $a b$ initio MP2/6-31G* y los resultados experimentales a 573 $\mathrm{K}$ :

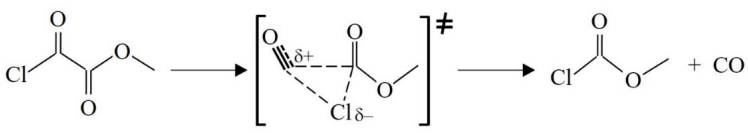

Figura 1. Termólisis de clorooxoacetato de metilo a $573 \mathrm{~K}$.

El método estático implica un sistema reactor cerrado en el cual se mide la presión total del gas generado conforme avanza la reacción a volumen constante, en combinación con el análisis químico de los productos a la temperatura de trabajo. Partiendo de todas estas consideraciones, se pretende describir el sistema estático en el cual van a ser obtenidos los parámetros cinéticos y termodinámicos de la termólisis del clorooxoacetato de etilo que permitan sugerir mecanismo para esta reacción, y hacer una comparación con lo reportado para el oxoacetato de etilo [12], otro éster derivado del ácido oxálico.

\section{Materiales y Métodos}

Línea de vacío. Elaborada con vidrio Pyrex y compuesta por un reactor cilíndrico, también de vidrio Pyrex (figura 2). El reactor permanece dentro de un horno con temperatura regulada y unido al manómetro y a la membrana de vidrio sensible a los cambios de presión. Los productos son recolectados en trampas de vidrio Pyrex a la temperatura del nitrógeno líquido. El sistema está acoplado a una bomba rotatoria de vacío HITACHI LTD 3VP-C2, con desplazamiento de aire de $50 \mathrm{~L} \mathrm{~min}^{-1}$, que genera un vacío de $6,67 \times 10^{-4}$ mbar. Para lograr vacío adicional, dispone también de una bomba difusora de mercurio de $150 \mathrm{~W}$.

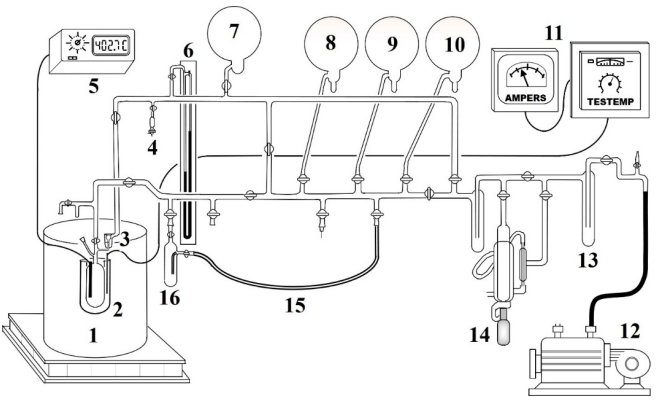

Figura 2. Sistema de vacío estático. 1, horno. 2, reactor. 3 , membrana de vidrio. 4, válvula compensadora. 5 , multímetro digital. 6, manómetro de mercurio. 7, matraz al vacío. 8-10, contenedores de $\mathrm{CO}_{2}$, propileno y etileno, respectivamente. 11 , control de temperatura. 12 , bomba de vacío. 13, trampa de recolección de producto gaseoso. 14 , bomba difusora de mercurio. 15, conexión de goma.

16 , recolector de producto para análisis.

Membrana de vidrio. Superficie reflectora de luz constituida por lámina de vidrio de $0,5 \mathrm{~mm}$ de espesor recubierta de una fina película de aluminio, insertada en diafragma y conectada, por su parte inferior, al reactor mediante un capilar, y al manómetro por su parte superior (figura 3). Es iluminada por una lámpara que produce reflexión de una línea indicadora, permitiendo fijar un punto de equilibrio antes de iniciarse la reacción. Este cero relativo corresponde a la igualdad de presión a ambos lados de la membrana. El incremento de presión en el sistema debido a la termólisis origina deformación en la membrana y pone en movimiento la línea indicadora respecto al punto de equilibrio. Es medido mediante la introducción de aire en la parte superior de la membrana con ayuda de la válvula compensadora, hasta llevar nuevamente la línea indicadora a la posición de referencias, siendo entonces la altura manométrica alcanzada equivalente a la presión total interna del reactor.

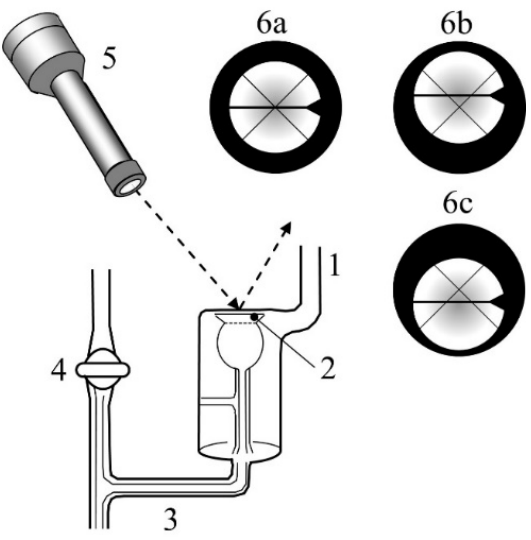

Figura 3. Sistema óptico. 1, conexión al manómetro. 2, membrana de vidrio. 3 , capilar. 4 , llave de paso al reactor. 5, lámpara. 6, visor con línea indicadora: a, en posición de equilibrio; b, con incremento de presión en el reactor; c, con vacío. 
3. Horno con regulador de temperatura. Contiene al reactor y está recubierto por camisa de calentamiento de $220 \mathrm{~V}$ y $800 \mathrm{~W}$, (figura 4), fijado sobre ladrillos refractarios. La variación máxima de temperatura es de $\pm 0,2 \mathrm{~K}$ y se mantiene constante por un controlador OMEGA SSR240AC45, acoplado a termocupla de hierroconstatan e insertado en un orificio dentro del bloque metálico. La temperatura de trabajo es medida con termopar de hierro-constantan conectado al multímetro digital.

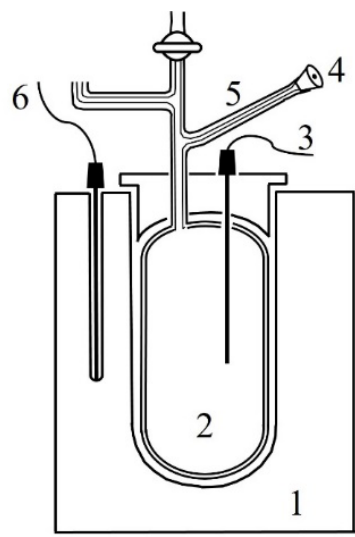

Figura 4. Corte longitudinal del horno. 1, bloque metálico. 2, reactor. 3 , termopar. 4 , punto de inyección con tapón de silicona. 5, capilar. 6 , termocupla.

\section{Obtención de los datos cinéticos}

Las mediciones se llevaron a cabo dentro del rango de 543-593 K y 76-209 mbar. Luego de hacer vacío en el sistema, se cierra la llave de paso para aislar el reactor. Aproximadamente $0,20 \mathrm{~mL}$ del sustrato fueron inyectados al reactor con una jeringa PERFEKTUM® a través del tapón de silicona. A intervalos adecuados de tiempo se mide el incremento de presión generado por los productos gaseosos en el reactor. La presión inicial de sustrato en el reactor P0 se determina por extrapolación a tiempo cero de una curva de presión total en función del tiempo. Cuando se requiere de aditivos tales como inhibidores de radicales libres y estándares, éstos se inyectan primero, se equilibra la presión del reactor $y$, seguidamente, se inyecta el sustrato. Los productos son sometidos a análisis cromatográfico en fase gaseosa y/o liquida, dependiendo de su estado físico, a temperatura ambiente. Al lograrse el porcentaje de reacción necesario, son extraídos de la cámara de reacción y condensados a la temperatura del nitrógeno líquido en las trampas de vidrio PYREX conectadas al sistema de vacío, a las cuales se les extrae previamente el aire. Una vez alcanzada la temperatura ambiente, cada muestra es inyectada al cromatógrafo hasta obtener resultados reproducibles.

\section{Caracterización del sustrato}

La identificación del clorooxoacetato de etilo
(Acros Organics, CAS № 4755-77-5), cuya pureza era de $98 \%$, fue verificada mediante GC/MS [12].

\section{Caracterización de los productos de reacción}

1. Análisis cromatográfico de la fase gaseosa. El etileno producido fue cuantificado en un cromatógrafo VARIAN $3700 \mathrm{GC}$ acoplado a integrador VARIAN $4400 \mathrm{con}$ detector FID (15 mL min-1 de $\mathrm{H}_{2}$ y $120 \mathrm{~mL} \mathrm{~min}^{-1}$ de aire) y columna empacada de PORAPAK Q 80/100 mesh $(3,1 \mathrm{~m}$ de longitud), con $\mathrm{N}_{2}\left(30 \mathrm{~mL} \mathrm{~min}^{-1}\right.$ ) y propileno (Matheson, Gas Products, Inc.) como gases de arrastre y estándar interno respectivamente. La temperatura del horno era de 373 K, y la ecuación obtenida de la curva de calibración fue la siguiente:

$$
P_{\text {etilend }} / P_{\text {propileno }}=1,556\left(A_{\text {etilend }} / A_{\text {propilend }}\right)-0,045
$$

2. Análisis cromatográfico de la fase líquida. El cloroformato de etilo, producto principal observado en la termólisis, fue analizado en un cromatógrafo HEWLETTPACKARD 5710-A acoplado a integrador HP 3392-A y equipado con un detector FID $\left(30 \mathrm{~mL} \mathrm{~min}^{-1} \mathrm{de}_{2}\right.$ y $240 \mathrm{~mL}$ $\mathrm{min}^{-1}$ de aire), con columna empacada de $10 \%$ SP 1200 , $1 \% \mathrm{H}_{3} \mathrm{PO}_{4}$, Chrom WAW 80/100 mesh (2 m de longitud), siendo la temperatura del horno igual a $313 \mathrm{~K}$, con $\mathrm{N}_{2}$ como gas de arrastre $\left(30 \mathrm{~mL} \mathrm{~min}^{-1}\right)$ y acetona p.a. (Merck, CAS № 67-64-1) como estándar interno. Obteniéndose la siguiente ecuación para la curva de calibración:

$$
P_{\text {CICOCOOEt }} / P_{\text {acetona }}=1,556\left(A_{\text {CICOCOOEt }} / A_{\text {acetona }}\right)-0,045
$$

\section{Determinación del coeficiente de velocidad}

Entre los productos de esta reacción fueron identificados etileno, $\mathrm{CO}_{2}$ y $\mathrm{ClCOOC}_{2} \mathrm{H}_{5}$, lo que sugiere podrían estar involucradas las siguientes vías de reacción:

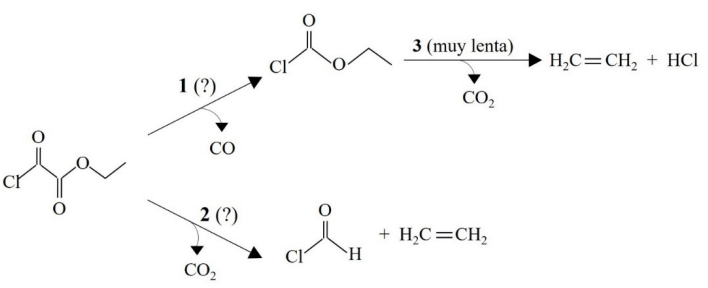

Figura 5. Posibles vías de descomposición térmica del clorooxoacetato de etilo.

El coeficiente de velocidad reportado para la termólisis de $\mathrm{ClCOOC}_{2} \mathrm{H}_{5}$ [13] obedece a la siguiente ecuación entre 559 y $626 \mathrm{~K}$ :

$$
k_{\mathrm{CICOCOOEt}}\left(\mathrm{s}^{-1}\right)=1012,64 e^{-183600 \mathrm{~J} / 8,314 T(\mathrm{~K})}
$$

Una alternativa para verificar si en el rango de temperatura de trabajo (543-593 K) ocurrirían todas las reacciones mostradas en la figura 5 , es determinar la 
presión de etileno producido y comparar con la calculada para la hipotética reacción 3. De ser iguales, sólo ocurre una vía: decarbonilación del sustrato, 1, cuyo intermediario genera etileno al descomponerse. Si la presión de etileno hallada por análisis cromatográfico es mayor que la del etileno proveniente de la pirólisis de $\mathrm{ClCOOC}_{2} \mathrm{H}_{5}$, significa que están ocurriendo dos reacciones paralelas y el etileno restante se generó de la decarboxilación, 2. La siguiente expresión permite determinar la presión de etileno producido de la pirólisis de $\mathrm{ClCOOC}_{2} \mathrm{H}_{5}$ a cualquier tiempo $t$, siendo la presión de $\mathrm{ClCOOC}_{2} \mathrm{H}_{5}$ residual obtenida de la ecuación (2).

$$
P_{\text {etileno generado de CICOOEt }}=P_{\mathrm{CICOOEt} \text { residual }}\left(\frac{1-e^{k_{\mathrm{CICOOEt}} t}}{e^{k_{\mathrm{CICOOE} t} t}}\right)(4)
$$

\section{Resultados y Discusión}

Estequiometría. Los productos que se presentan en el esquema de la figura 5 fueron encontrados en todo el rango de temperatura de trabajo. El CO no es licuable a la temperatura del nitrógeno líquido, por lo que su detección se logró a través de un generador de alto voltaje [12]. La tabla 1 muestra una comparación de los resultados teóricos obtenidos de la ecuación (4) para la presión de etileno que esperaría obtenerse de la termólisis de $\mathrm{ClCOOC}_{2} \mathrm{H}_{5}$, con la presión de etileno realmente obtenida de la ecuación (1). La diferencia entre estos valores a los respectivos tiempos de reacción para cada temperatura, es indicio de que el etileno identificado proviene realmente de la descomposición de $\mathrm{ClCOOC}_{2} \mathrm{H}_{5}, \mathrm{y}$ no ocurre paralelamente una reacción de decarboxilación como en la termólisis del oxoacetato de etilo.

Tabla 1. Comparación entre etileno total analizado en termólisis de clorooxoacetato de etilo y etileno generado en termólisis de cloroformato de etilo a diferentes temperaturas.

\begin{tabular}{llccc}
\hline \multirow{2}{*}{$T(\mathrm{~K})$} & \multicolumn{2}{c}{ Parámetro } & \multicolumn{3}{c}{ Valor } \\
\hline \multirow{3}{*}{563,2} & $t$ (min) & 20 & 30 & 40 \\
& $P_{\text {etileno, GC }}(\mathrm{mbar})$ & 0,19 & 0,29 & 0,27 \\
& $P_{\text {etileno generado de CICOOEt }}(\mathrm{mbar})$ & 2,00 & 2,93 & 3,60 \\
& $t$ (min) & 12 & 15 & 20 \\
573,2 & $P_{\text {etileno, GC }}(\mathrm{mbar})$ & 2,53 & 3,07 & 3,73 \\
& $P_{\text {etileno generado de CICOOEt }}(\mathrm{mbar})$ & 2,40 & 2,93 & 3,87 \\
& $t$ (min) & 2 & 3 & 6 \\
593,1 & $P_{\text {etileno, GC }}$ (mbar) & 0,67 & 1,47 & 3,73 \\
& $P_{\text {etileno generado de CICOOEt }}(\mathrm{mbar})$ & 0,80 & 1,60 & 4,00 \\
\hline
\end{tabular}

La presión de etileno producido es baja porque en el rango de trabajo la termólisis de $\mathrm{ClCOOC}_{2} \mathrm{H}_{5}$ es muy lenta. Por otra parte, aunque no se llevó a cabo análisis de $\mathrm{HCl}$ pudo detectarse la presencia de $\mathrm{CO}$ en la línea de vacío. Estas observaciones permiten sugerir la siguiente secuencia de reacciones para la descomposición térmica del clorooxoacetato de etilo:

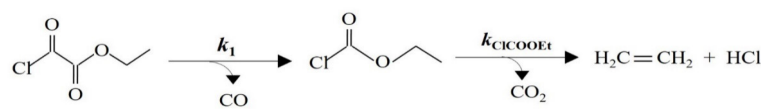

Figura 6. Reacciones identificadas en la pirólisis de clorooxoacetato de etilo.

En la tabla 2 puede apreciarse la similitud entre los porcentajes de reacción determinados por diferentes métodos, lo que sustenta la estequiometria propuesta. Para cada tiempo de reacción, fueron obtenidos a partir de las siguientes ecuaciones, donde $P_{0}$ es la presión inicial del sustrato en el reactor:

$$
\% \text { Reacción(método manométrico) }=100\left[\left(P_{\text {tota }}\left(P_{0}\right)-1\right]\right.
$$

$$
\% \text { Reacción (método cromatográfico) }=100\left[\left(P_{\mathrm{CICOCOOE}}\left(P_{0}\right)\right](6)\right.
$$

Tabla 2. Verificación de estequiometría en la termólisis de cloroxoacetato de etilo a 573,2 K.

\begin{tabular}{crrrrr}
\hline Parámetro & \multicolumn{5}{c}{ Valor } \\
\hline$t$ (min) & 7 & 10 & 12 & 15 & 20 \\
$\begin{array}{c}\text { \%Reacción (método } \\
\text { cromatográfico) }\end{array}$ & 26,2 & 33,5 & 41,0 & 46,8 & 54,8 \\
$\begin{array}{c}\text { \%Reacción (método } \\
\text { manométrico) }\end{array}$ & 26,9 & 34,6 & 40,4 & 46,8 & 56,4 \\
\hline
\end{tabular}

Orden de reacción. La correlación lineal mostrada en la figura 7 permite sugerir una cinética de orden uno en todo el rango de temperaturas de trabajo. De igual forma que para la termólisis del oxoacetato de etilo, cuya reacción de decarbonilación resultó ser de primer orden.

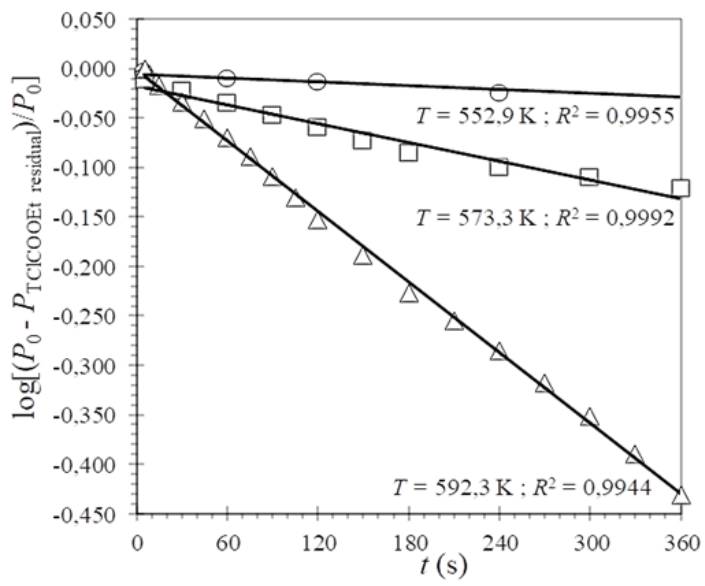

Figura 7. Gráfica de orden uno para la termólisis de clorooxoacetato de etilo. 
Los valores del coeficiente de velocidad fueron obtenidos de la siguiente ecuación:

$$
k_{1}=-(2,303 / t) \log \left[\left(P_{0}-P_{\mathrm{ClCOOEt}}\right) / P_{0}\right]
$$

Efecto del inhibidor. Para impedir que la reacción siguiese un mecanismo por radicales libres en cadena [14-18] en lugar de la vía molecular, se utilizó tolueno como inhibidor de radicales [20-25] en diferentes proporciones. En la tabla 3 puede observarse cómo la invariabilidad del coeficiente de velocidad en presencia y ausencia de tolueno es indicio de que esta reacción en estudio no estuvo inhibida, lo que permite sugerir un mecanismo molecular.

Tabla 3. Efecto del tolueno como inhibidor sobre el coeficiente de velocidad en la termólisis de clorooxoacetato de etilo a $582,5 \mathrm{~K}$

\begin{tabular}{cccccc}
\hline $\begin{array}{c}\text { Presión de } \\
\text { inhibidor, } \\
P_{\mathrm{i}} \text { (mbar) }\end{array}$ & $\begin{array}{c}\text { Presión } \\
\text { inicial, } \\
P_{0} \text { (mbar) }\end{array}$ & $\begin{array}{c}\text { Relación } \\
P_{\mathrm{i}} / P_{0}\end{array}$ & $\begin{array}{c}10^{4} k_{1} \\
\left(\mathrm{~s}^{-1}\right)\end{array}$ & $\begin{array}{c}\text { Promedio } \\
<10^{4} k_{1}> \\
\left(\mathrm{s}^{-1}\right)\end{array}$ & $\begin{array}{c}\text { DSR } \\
(\%)\end{array}$ \\
\hline & 111,99 & - & 7,10 & & \\
119,99 & 98,66 & 1,22 & 6,97 & & \\
183,32 & 93,33 & 1,96 & 6,88 & $6,99 \pm 0,09$ & 1,29 \\
258,65 & 83,99 & 3,08 & 6,99 & & \\
\hline
\end{tabular}

Homogeneidad. Para verificar que la termólisis ocurriese cuantitativamente en fase gas [26-28], se realizó adicionalmente en un reactor empacado internamente con pequeños cilindros de vidrio Pyrex con relación superficie-volumen de $6,22 \mathrm{~cm}^{-1}$ (figura 8), asumiéndose esta relación igual a la unidad para el reactor no empacado (figura 4). Sólo hubo consistencia de resultados cuando ambos reactores fueron recubiertos internamente con una capa de carbón producto de la termólisis de bromuro de alilo sobre $673 \mathrm{~K}$ [29]. No fue posible obtener resultados reproducibles en los reactores sin recubrimiento de carbón, lo que revela que la naturaleza de la superficie de vidrio puede afectar significativamente la velocidad de reacción [30].
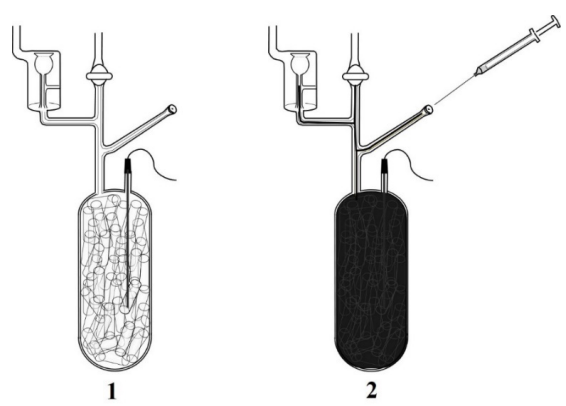

Figura 8. Reactor de vidrio Pyrex empacado: 1, limpio. 2, recubierto de carbón producido en la termólisis a $673 \mathrm{~K}$ luego de inyectar bromuro de alilo.
Los resultados indican que la reacción es homogénea bajo tales condiciones.

Tabla 4. Homogeneidad de la descomposición térmica del clorooxoacetato de etilo a 573,2 K.

\begin{tabular}{cc}
\hline \multicolumn{2}{c}{$10^{4} k_{1}\left(\mathrm{~s}^{-1}\right)$} \\
\hline $\begin{array}{c}\text { Reactor no empacado } \\
\left(\mathrm{S} / \mathrm{V}=1 \mathrm{~cm}^{-1}\right)\end{array}$ & $\begin{array}{c}\text { Reactor empacado } \\
\left(\mathrm{S} / \mathrm{V}=6,22 \mathrm{~cm}^{-1}\right)\end{array}$ \\
$7,05 \pm 0,16$ & $7,70 \pm 0,38$ \\
\hline
\end{tabular}

\section{Efecto de la temperatura}

Los parámetros cinéticos fueron obtenidos por regresión lineal de los siguientes resultados. La figura 9 corresponde al gráfico de Arrhenius.

Tabla 5. Datos de la gráfica de Arrhenius para la termólisis de clorooxoacetato de etilo.

\begin{tabular}{rrrrrrr}
\hline$T(\mathrm{~K})$ & 543,2 & 552,9 & 563,2 & 573,2 & 583,3 & 593,1 \\
\hdashline $10^{4} k_{1}\left(\mathrm{~s}^{-1}\right)$ & 1,01 & 1,80 & 3,82 & 7,00 & 13,47 & 29,36 \\
\hline
\end{tabular}

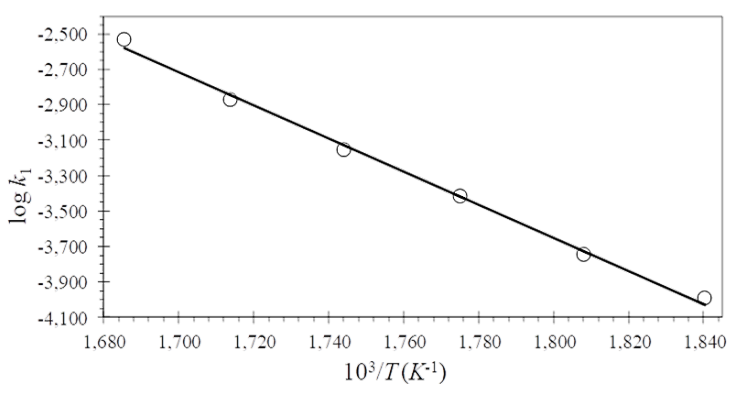

Figura 9. Influencia de la temperatura sobre el coeficiente de velocidad de reacción de clorooxoacetato de etilo. Pendiente: $-(9371,9 \pm 256,0)$ K. Intercepto: $(13,22 \pm 0,45) \cdot R^{2}=0,9990 \pm 0,0333$. Nivel de confianza: $90 \%$

Así, se obtuvo la ecuación de Arrhenius para la reacción entre 543,2 - 593,1 K:

$$
\log k_{1}=(13,22 \pm 0,45)-(179,4 \pm 4,9) \mathrm{kJ} \mathrm{mol}^{-1}(2,303 R T)^{-1}(8)
$$

Con base en la teoría del estado de transición, es posible determinar los parámetros cinéticos y termodinámicos de activación, mediante las siguientes ecuaciones para reacciones unimoleculares:

$$
\begin{gathered}
\Delta G^{\neq}=\Delta H^{\neq}-T \Delta S^{\neq} \\
\Delta H^{\neq}=E_{\mathrm{a}}-R T
\end{gathered}
$$




$$
\Delta S^{\neq}=R \ln \left(\frac{A h}{e k_{\mathrm{B}} T}\right)
$$

Los resultados obtenidos por extrapolación a 623 $\mathrm{K}$ se presentan en la tabla 6 . Se escogió esta temperatura para poder incluir los resultados de la decarbonilación del oxoacetato de etilo, con el fin de discutir el efecto del sustituyente al grupo carbonilo sobre la reactividad.

Tabla 6. Comparación entre los parámetros cinéticos obtenidos a $623 \mathrm{~K}$ en las reacciones de decarbonilación de oxoacetato de etilo y clorooxoacetato de etilo.

\begin{tabular}{cccccccc}
\hline $\mathrm{Z}^{\mathrm{a}}$ & $\begin{array}{c}10^{4} k_{\mathrm{r}} \\
\left(\mathrm{s}^{-1}\right)^{2}\end{array}$ & $\log A$ & $\begin{array}{c}E_{\mathrm{a}} \\
\left(\mathrm{kJ} \mathrm{mol}^{-1}\right)\end{array}$ & $\begin{array}{c}\Delta H \neq \\
\left(\mathrm{kJ} \mathrm{mol}^{-1}\right)\end{array}$ & $\begin{array}{c}\mathrm{DS} \neq \\
\left(\mathrm{J} \mathrm{mol}^{-1} \mathrm{~K}^{-1}\right)\end{array}$ & $\begin{array}{c}\Delta G \neq \\
\left(\mathrm{kJ} \mathrm{mol}^{-1}\right)\end{array}$ & Ref. \\
\hline $\mathrm{Cl}$ & 151,57 & $\begin{array}{c}13,22 \\
\pm 0,45\end{array}$ & $\begin{array}{c}179,4 \pm \\
4,9\end{array}$ & 227,7 & $-6,3$ & 178,1 & $\mathrm{~b}$ \\
$\mathrm{H}$ & 0,034 & $\begin{array}{c}14,06 \\
\pm 0,54\end{array}$ & $\begin{array}{c}232,9 \pm \\
7,0\end{array}$ & 174,2 & 9,8 & 221,6 & 9 \\
\hline \multicolumn{7}{c}{} \\
\end{tabular}

${ }^{\text {aS }}$ ustituyente en la parte acílica del éster

${ }^{\mathrm{b}}$ Este trabajo

Con base en estos resultados, se sugiere un mecanismo que involucra reacciones de decarbonilación y eliminación de etileno. En este caso, se trata de reacciones consecutivas, a diferencia de la termólisis de oxoacetato de etilo, donde ocurren reacciones paralelas.

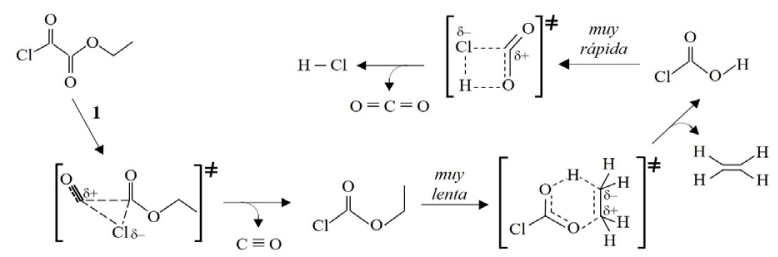

Figura 10. Mecanismo para la descomposición térmica de clorooxoacetato de etilo.

En ambos casos, los valores positivos tanto de entalpía como de energía libre de activación sugieren estados de transición endotérmicos y endergónicos. El valor negativo de entropía de activación en la decarboxilación del clorooxoacetato de etilo puede atribuirse a la formación de un enlace triple en el estado de transición, lo que disminuye los grados de libertad. En contraste con la reacción de decarbonilación del oxoacetato de etilo, cuyo signo positivo de la entropía de activación puede ser debido a que el hidrógeno acílico forme enlaces menos rígidos en el estado de transición dado su comportamiento dual, como ácido y nucleófilo. El sustituyente cloro parece asistir la reacción de decarbonilación preferentemente sobre la reacción de eliminación. Este comportamiento puede ser razonado en términos de trasmisión electrónica: el átomo de cloro es buen "saliente" y dispone de pares electrónicos no enlazantes. Estas propiedades le permiten migrar y atacar nucleofílicamente la densidad de carga positiva que se genera en el átomo de carbono del grupo carbonilo vecino en el estado de transición. Este comportamiento sugiere que mientras más electronegativo es el sustituyente, mayor es su tendencia a migrar al carbonilo vecino porque tiende a atraer la densidad electrónica del carbonilo vecino a medida que se va debilitando el enlace entre los grupos carbonilo adyacentes en el estado de transición, tal como se muestra en la figura 10 . Sobre esta base puede explicarse el hecho de que el coeficiente de velocidad de decarbonilación del clorooxoacetato de etilo resulte ser drásticamente mayor que el oxoacetato de etilo.

\section{Conclusiones}

En base al análisis cualitativo-cuantitativo de productos y los parámetros cinéticos obtenidos, es posible proponer un mecanismo de decarbonilación, con reacciones consecutivas de decarboxilación y eliminación de etileno, para la eliminación unimolecular y homogénea del clorooxoacetato de etilo. La entropía de activación del clorooxoacetato de etilo es negativa porque el cloro tiene una marcada tendencia de migrar al carbonilo contiguo, formando enlaces con menos grados de libertad en el estado de transición. La tendencia que muestra un sustituyente de migrar al grupo carbonilo adyacente en reacciones de decarbonilación parece ser directamente proporcional a su electronegatividad. A una misma temperatura, la reacción de decarbonilación del clorooxoacetato de etilo resultó ser tres órdenes de magnitud superior a la decarbonilación del oxoacetato de etilo.

\section{Agradecimiento}

Al Instituto Venezolano de Investigaciones Científicas (IVIC), muy especialmente a todo el grupo de trabajo del Laboratorio de Fisicoquímica Orgánica.

\section{Referencias Bibliográficas}

[1] Chuchani G., Martin I. y Avila I.: "Effect of substituents in the gas- phase elimination kinetics of $\beta$ - substituted ethyl acetates". Int. J. Chem. Kinet., Vol 11, № 6 (1979) 561-567. https://doi. org/10.1002/kin.550110602

[2] Al Awadi N.A., Kaul K. y El Dusouqui O.M.: "Kinetics and mechanism of thermal gas- phase elimination of $\alpha$ substituted carboxylic acids: role of relative basicity of $\alpha$-substituents and acidity of incipient proton". J. Phys. Org. Chem., Vol 13, № 9 (2000) 499-504. https://doi.org/10.1002/10991395(200009)13:9<499::AID-POC269>3.0.CO;2-0

[3] Al-Awadi S.A., Abdallah M.R., Dib H.H., Ibrahim 
M.R., Al-Awadi N.A. y El-Dusouqui O.M.E.: "Kinetics and mechanism of thermal gas-phase elimination of $\beta$-substituted carboxylic acids". Tetrahedron, Vol. 61, № 24 (2005), 5769-5777. https://doi. org/10.1016/j.tet.2005.04.031

[4] Silva A.M.: "A theoretical study of the pyrolysis of isopropyl acetate". Chem. Phys. Lett., Vol. 439, № 1-3 (2007) 8-13. https://doi.org/10.1016/j. cplett.2007.03.037

[5] Floyd K. y Smith G.: "Structure-reactivity relationships in homogeneous gas phase reactions: thermolysis and rearrangements". Prog. Phys. Org. Chem., Vol. 8 (2009) 75. https://10.1002/9780470171875.ch2

[6] Moldoveanu S.C.: "The chemistry of the pyrolytic process". In Techniques and Instrumentation in Analytical Chemistry, Amsterdam, Elsevier, Vol 28 (2010) 28:7-48. https://doi.org/10.1016/S01679244(09)02802-9

[7] Wu P., Chen X., Li J. y Huang Y.: "Theoretical studies on the pyrolysis of thiocarbonates". Comput. Theor. Chem., Vol. 1030 (2014) 67-73. https://doi. org/10.1016/j.comptc.2013.12.026

[8] Shiroudi A. y Zahedi E.: "Understanding the kinetics of thermal decomposition of 2, 3-epoxy-2, 3-dimethylbutane using RRKM theory". RSC Adv., Vol. 6, № 94 (2016) 91882-91892. https://doi. org/10.1039/C6RA21963B

[9] Al-Awadi N.: "Gas-Phase Pyrolytic Reactions. Chapter 4: Structure/Reactivity Correlation" (2019) https://doi.org/10.1002/9781119010753.ch4

[10] Laidler K.J.: "Reaction kinetics: Homogeneous gas reactions". Vol. 1. Amsterdam, Elsevier (2013) 97149.

[11] Cordova T., Rotinov A.y Chuchani G.: "Experimental and theoretical study of the homogeneous, unimolecular gas-phase elimination kinetics of methyl oxalyl chloride". J. Phys. Org. Chem. Vol 17, № 2 (2004) 148-151. https://doi.org/10.1002/ poc.705

[12] Reyes Y.A.: "Estudio cinético de la termólisis del oxoacetato de etilo". Afinidad, Vol. 74, № 579 (2017) 208-213. https://www.raco.cat/index. php/afinidad/article/view/328556

[13] Johnson R. L. y Stimson V.R.: "The thermal decomposition of ethyl chloroformate". Aust. J. Chem., Vol. 29, № 6 (1976) 1389-1392. https:// doi.org/10.1071/CH9761389
[14] Benson, S.W.: "Predictability of chain reactions". Ind. Eng. Chem., Vol. 56, № 1 (1964) 18-27. https://doi.org/10.1021/ie50649a004

[15] Márquez E., Domínguez R., Tosta M. y Chuchani, G.: "Cinética y mecanismos de la eliminación homogénea en fase gaseosa unimolecular del ortoacetato de trimetilo y del ortobutirato de trimetilo". J. Phys. Chem. A, Vol. 112, № 47 (2008) 12140-12142. https://doi.org/10.1021/ jp8074942

[16] Vasiliou A.G., Piech K.M., Zhang X., Nimlos M.R., Ahmed M., Golan A. et al.: "The products of the thermal decomposition of $\mathrm{CH}_{3} \mathrm{CHO}$. J. Chem. Phys., Vol. 131, № 1 (2011) 014306. https://doi. org/10.1063/1.3604005

[17] Moreno B.M, Quach A.L., Merves M.N. y Klein M.T.: "Discrimination between free-radical and concerted pyrolysis mechanisms". Energy Fuels, Vol. 28, № 7 (2014) 4256-4259. https://doi. org/10.1021/ef500055a

[18] Trubetskaya A., Jensen P.A., Jensen A.D., Glarborg P., Larsen F.H. y Andersen M.L.: "Characterization of free radicals by electron spin resonance spectroscopy in biochars from pyrolysis at high heating rates and at high temperatures". Biomass Bioenergy, Vol. 94 (2016) 117-129. https://doi. org/10.1016/j.biombioe.2016.08.020

[19] Vahid S. y Zokaie M.: "Multichannel Gas-Phase Unimolecular Decomposition of Acetone: Theoretical Kinetic Studies". J. Phys. Chem. A, Vol. 122, № 28 (2018) 5895-5904. https://doi. org/10.1021/acs.jpca.8b02423

[20] Colket M.B. y Seery D.J. (1994, January).: "Reaction mechanisms for toluene pyrolysis". In Symposium (International) on combustion (Vol. 25, No. 1, pp. 883-891). Elsevier. https://doi.org/10.1016/ S0082-0784(06)80723-X

[21] Sivaramakrishnan R., Tranter R.S. y Brezinsky K.: "High pressure pyrolysis of toluene. 1. Experiments and modeling of toluene decomposition". J. Phys. Chem A., Vol. 110, № 30 (2006) 9388-9399. https://doi.org/10.1021/jp060820j

[22] Sivaramakrishnan R., Tranter R.S. y Brezinsky K.: "High pressure pyrolysis of toluene. 2. Modeling benzyl decomposition and formation of soot precursors". J. Phys. Chem. A, Vol. 110, № 30 (2006) 9400-9404. https://10.1021/jp0608224

[23] Zhang L., Cai J., Zhang T. y Qi F.: "Kinetic modeling study of toluene pyrolysis at low pressure". Combust. Flame, Vol. 157, № 9 (2010) 1686-1697. 
https://10.1016/j.combustflame.2010.04.002

[24] Lannuzel F., Bounaceur R., Michels R., Scacchi G. y Marquaire P.M.: "An extended mechanism including high pressure conditions (700bar) for toluene pyrolysis”. J. Anal. Appl. Pyrol., Vol. 87, № 2 (2010) 236-247. https://10.1016/j.jaap.2010.01.001

[25] Matsugi A. y Miyoshi A.: "Modeling of two- and three-ring aromatics formation in the pyrolysis of toluene". Proc. Comb. Inst., Vol. 31, № 1 (2013) 269-277. https://10.1016/j.proci.2012.06.032

[26] Taylor J.E. y Milazzo T.S.: "Gas phase pyrolysis of 2,2,3,3 tetramethylbutane using a wall less reactor". Int. J. Chem. Kinet., Vol. 10, № 12 (1978) 12451257. https://doi.org/10.1002/kin.550101207

[27] Dabbagh H.A. y Davis B.H.: "Pyrolysis of sec-butyl acetate. Is the stereospecific syn elimination a homogeneous or heterogeneous reaction?". J. Org. Chem., Vol. 55, № 7 (1990) 2011-2016. https:// doi.org/10.1021/jo00294a011

[28] Mascavage L.M. y Dalton D.R.: "Surface catalyzed hydrochlorination of 1,3-butadiene". Tetrahedron Lett., Vol. 32, № 29 (1991) 3461-3464. https:// doi.org/10.1016/0040-4039(91)80806-H

[29] Maccoll A.: "Studies in the pyrolysis of organic bromides. Part I. The kinetics of the decomposition of allyl bromide". J. Chem. Soc. (1955) 965-973. https://doi.org/10.1039/JR9550000965

[30] Acevedo S., Galicia L., Plaza E., Atencio R., Rodríguez A. y González E.: "Carbón activado preparado a partir de carbón mineral bituminoso activado con hidróxido de potasio". Rev. Téc. Ing. Univ. Zulia. Vol. 39, No 2 (2016) 64-70. https://www. produccioncientificaluz.org/index.php/tecnica/ article/view/21324 


\section{REVISTA TECNICA}

DE LA FACULTAD DE INGENIERIA

UNIVERSIDAD DEL ZULIA

Vol. 43. $\mathrm{N}^{\circ} 3$, Septiembre - Diciembre 2020 pp. 114 - 176

Esta revista fue editada en formato digital y publicada el 31 de Agosto de 2020, por el Fondo Editorial Serbiluz, Universidad del Zulia. Maracaibo-Venezuela

www.luz.edu.ve

www.serbi.luz.edu.ve

www.produccioncientifica.org 